\title{
High Erythrocyte Sedimentation Rate is Associated with White Matter Hyperin- tensity in a Healthy Population
}

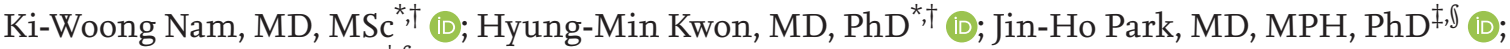 \\ Hyuktae Kwon, MD, $\mathrm{PhD}^{\ddagger, \Phi}$ (D) \\ Department of Neurology, Seoul National University College of Medicine ${ }^{\star}$, Seoul; Department of Neurology, Seoul Metropolitan Government- \\ Seoul National University Boramae Medical Center ${ }^{\dagger}$, Seoul; Department of Family Medicine, Seoul National University College of Medicine ${ }^{\ddagger}$, \\ Seoul; Department of Family Medicine, Seoul National University Hospital ${ }^{\mathbb{1}}$, Seoul, Korea
}

Background: High erythrocyte sedimentation rate (ESR) is related to various metabolic, cardiovascular, and cerebrovascular diseases; however, there is a limited study about its association with subclinical cerebral small vessel diseases (cSVDs). In this study, we evaluated the relationship between ESR and cSVDs in a healthy population.

Methods: We evaluated healthy check-up volunteers between 2006 and 2013. As subtypes of cSVD, we evaluated white matter hyperintensity (WMH), silent brain infarct (SBI), and cerebral microbleeds (CMBs). The WMH volume was rated quantitatively using a computer-assisted semi-automated technique. SBI and CMBs were evaluated qualitatively for presence.

Results: A total of 2,553 participants were evaluated. In multivariable linear regression analysis, $\operatorname{ESR}(\beta=0.027,95 \%$ confidence interval $[\mathrm{CI}]=0.001$ to $0.053, p=0.043)$ was significantly associated with WMH volumes after adjusting for confounders. Age $(\beta=0.046,95 \% \mathrm{CI}=0.042$ to $0.051, p<0.001)$, hypertension $(\beta=0.187,95 \%$ $\mathrm{CI}=0.092$ to $0.281, p<0.001)$, and white blood cell counts $(\beta=0.042,95 \% \mathrm{CI}=0.018$ to $0.067, p=0.001$ ) were also closely related to WMH volumes. ESR did not show any statistical significance with SBI or CMBs. In our data, ESR were closely related to age, sex, diabetes, hyperlipidemia, current smoking, systolic and diastolic blood pressure, and high-sensitivity C-reactive protein.

Conclusion: High ESR was associated with larger WMH volumes in a healthy population. Since this close association was not seen in SBI or CMBs, WMH may be more related to inflammation-related pathologies.

\section{J Neurosonol Neuroimag 2021;13(1):7-13}

Key Words: Leukoaraiosis; Inflammation; Cerebral small vessel diseases; Erythrocytes

\author{
Received: April 21, 2021 \\ Revised: May 11, 2021 \\ Accepted: May 25, 2021 \\ Correspondence: \\ Hyung-Min Kwon, MD, PhD \\ Department of Neurology, \\ Seoul Metropolitan Govern- \\ ment-Seoul National University \\ Boramae Medical Center, Seoul \\ National University College \\ of Medicine, 20 Boramae-ro \\ 5-gil, Dongjak-Gu, Seoul o7o61, \\ Korea \\ Tel: $+82-2-870-2475$ \\ Fax: $+82-2-831-2826$ \\ E-mail: hmkwon@snu.ac.kr
}

\section{INTRODUCTION}

Cerebral small vessel disease (cSVD) is a subclinical pathology, often found in the elderly. ${ }^{1,2}$ Although cSVDs themselves are asymptomatic, they are clinically important because they increase the risk of ischemic stroke or vascular dementia. ${ }^{3-5}$ There are subtypes with various pathologies (e.g., white matter hyperin- tensity [WMH], silent brain infarct [SBI], and cerebral microbleeds [CMBs]) in cSVD. These cSVD subtypes are very different in appearance, but are often found together in brain magnetic resonance imaging (MRI)., ${ }^{1,6}$ In addition, previous autopsy studies have also found a link between them. ${ }^{2,7}$ Therefore, many studies have been conducted to find the common pathological mechanisms shared between them. Various mecha- 
nisms have been proposed as hypotheses, and subclinical inflammation is one of them. .10 $^{-10}$

Erythrocyte sedimentation rate (ESR) is an index that reflects the aggregation of red blood cells, and is accelerated by the asymmetry of charged proteins (e.g., fibrinogen, immunoglobulin, lipoprotein) accompanying the occurrence of inflammation. ${ }^{11,12}$ Since ESR is an inexpensive, validated, and generally available test, it has long been used as a nonspecific inflammatory marker. ${ }^{11-13}$ In addition, ESR, like other inflammatory markers, has been closely associated with various metabolic diseases, atherosclerosis, cardiovascular diseases, and cerebrovascular diseases. ${ }^{14-18}$ It is thought that ESR will also have a close relationship with cSVD, which shares similar pathologies with these, but study on this has not been done yet.

In this study, we aimed to evaluate the relationship between ESR and cSVD in a neurologically healthy population. In addition, through comparisons between cSVD subtypes, we would like to examine whether ESR is involved in the common pathological mechanisms of cSVD development or whether it is more closely related to a specific cSVD subtype.

\section{SUBJECTS AND METHODS}

\section{Study population}

From a consecutive registry at a large medical center in Korea (Seoul National University Hospital Health Promotion Center), we included participants who visited to conduct a voluntary routine health check-up, between January 2006 and December $2013 .{ }^{19}$ Then, we additionally excluded participants with the following conditions: 1) age under 30 years, 2) history of stroke or severe neurological deficit, 3) hemato-oncologic conditions or use of immunosuppressant, 4) having major surgery or severe trauma, 5) severe hepatic or renal disease, or 6) active infection within prior 2 weeks. ${ }^{19}$ Finally, a total of 2,553 subjects were included in the final analysis.

This study was approved by the Institutional Review Board (IRB) of the Seoul National University Hospital (IRB No. 1502-026-647). The requirement to obtain informed consent from participants was waived by the IRB due to the retrospective study design using de-identified information. All experiments were performed in accordance with the Declaration of Helsinki and relevant guidelines and regulations.

\section{Demographic, clinical, and laboratory assessments}

We conducted broad evaluations including age, sex, hypertension (using anti-hypertensive agents, or $\geq 140 \mathrm{mmHg}$ systolic blood pressure [BP], or $\geq 90 \mathrm{mmHg}$ diastolic BP), diabetes (using glucose-lowering agents, or $\geq 6.5 \%$ hemoglobin A1c levels), hyperlipidemia (using lipid-lowering agents, or $\geq 240 \mathrm{mg} / \mathrm{dL}$ total cholesterol levels), ischemic heart disease, current smoking, systolic and diastolic BP, and use of anti-platelet agents. ${ }^{19}$ Blood pressure was checked after 5 minutes rest in sitting position. Laboratory examinations were conducted on the same day after 12 hours of overnight fasting, including glucose profiles, lipid profiles, white blood cell (WBC) counts, high-sensitivity C-reactive protein (hsCRP) and ESR. ${ }^{19}$

\section{Radiological assessments}

All participants underwent brain MRI and MRA using 1.5-T MR scanners (Signa, GE Healthcare, Milwaukee, WI, or Magnetom SONATA, Siemens, Munich, Germany). The detailed acquisitions of MRI were as follows: T1-weighted images (repetition time [TR]/echo time $[\mathrm{TE}]=500 / 11 \mathrm{~ms}), \mathrm{T} 2$-weighted images $(\mathrm{TR} / \mathrm{TE}=5,000 / 127$ $\mathrm{ms}), \mathrm{T} 2$-gradient echo images $(\mathrm{TR} / \mathrm{TE}=57 / 20 \mathrm{~ms}$ ), T2 fluid-attenuated inversion recovery images (TR/ $\mathrm{TE}=8,800 / 127 \mathrm{~ms}$ ), and three-dimensional time of flight MRA images (TR/TE=24/3.5 ms, slice thickness $=1.2 \mathrm{~mm}$ ). The slice thickness was $5 \mathrm{~mm}$, except for time of flight MRA images.

As subtypes of cSVD lesions, we measured WMH volumes and the presence of SBI and CMBs. WMH volumes were rated quantitatively using Medical Imaging Processing, Analysis, and Visualization (MIPAV, version 7.3.0, National Institutes of Health, Bethesda, MD, USA). ${ }^{19}$ In this process, we used a computer-assisted semi-automated technique, measuring from converted DICOM files, as in our previous studies. ${ }^{19,20}$ SBI was defined as an asymptomatic and well-defined lesion larger than $3 \mathrm{~mm}$ in size, with signal characteristics same as 
those of the cerebrospinal fluid on T1- or T2-weighted images. ${ }^{1}$ CMBs were defined as focal round lesions less than $10 \mathrm{~mm}$ in size with low signal on T2-gradient echo images. ${ }^{1}$ All radiological parameters were rated by two neurologists (K.W.N. and H.Y.J.), and disagreements were resolved by discussion with a third rater (H.M.K.).

\section{Statistical analysis}

All statistical analyses were performed using SPSS version 20.0 (IBM Corp., Armonk, NY, USA). Univariate linear regression analyses were conducted to identify possible predictors for WMH volumes. Continuous variables with skewed data were transformed into a squared root scale, including WMH volumes. Then, variables with $p<0.10$ were introduced into the multivariable linear regression analysis along with age and sex as confounders. Since SBI and CMBs are binary outcome variables, we used multivariable logistic regression analyses for both. In addition, we investigated the relationship between ESR and demographic, clini$\mathrm{cal}$, and laboratory parameters in order to understand the characteristics of subjects with high ESR. For this analysis, we used a simple linear regression analysis. All variables with a $p<0.05$ were considered statistically significant in this study.

\section{RESULTS}

A total of 2,553 participants were evaluated (mean age: $57 \pm 10$ years, male sex: $54 \%$ ). The mean WMH volumes were $2.53 \pm 6.06 \mathrm{~mL}$. SBI and CMB were found in 193 (7.6\%) and 108 (4.2\%), respectively. The mean ESR was $12 \pm 13 \mathrm{~mm} /$ hour. Other detailed baseline characteristics are shown in Table 1.

In univariate linear regression analysis, WMH volumes were significantly associated with age, hypertension, diabetes, hyperlipidemia, current smoking, systolic and diastolic BP, hemoglobin Arc levels, fasting glucose levels, total cholesterol levels, WBC counts, ESR, and use of anti-platelet agents (Table 2).

In multivariable linear regression analysis, ESR $(\beta=0.027,95 \%$ confidence interval $[\mathrm{CI}]=0.001$ to 0.053 , $p=0.043$ ) showed significant association with WMH volumes after adjusting for confounders. Age $(\beta=0.046,95 \%$
$\mathrm{CI}=0.042$ to $0.051, p<0.001)$, hypertension $(\beta=0.187,95 \%$ $\mathrm{CI}=0.092$ to $0.281, p<0.001)$, and $\mathrm{WBC}$ counts $(\beta=0.042$, $95 \% \mathrm{CI}=0.018$ to $0.067, p=0.001$ ) were also significantly associated with WMH volumes, being independent from ESR (Table 3). On the other hand, neither SBI nor CMBs showed statistical significance with ESR (Supplementary Table 1).

In our data, ESR were associated with older age, female sex, diabetes, hyperlipidemia, current smoking, diastolic BP, hemoglobin Arc level, fasting glucose level, total cholesterol level, and hs-CRP level (Table 4).

\section{DISCUSSION}

In this study, we found that high ESR were associated with larger WMH volumes in a healthy population. In addition, WMH volume was closely related to WBC counts, and ESR was not statistically significant with SBI and CMBs. Given these findings, WMH appears to

TABLE 1. Baseline characteristics (total $n=2,553$ )

\begin{tabular}{lc}
\hline Age (years) & $57 \pm 10$ \\
Sex (male) & $1,396(54.7)$ \\
\hline Body mass index $\left(\mathrm{kg} / \mathrm{m}^{2}\right)$ & $24.17 \pm 3.06$ \\
\hline Hypertension & $656(25.7)$ \\
\hline Diabetes & $404(15.8)$ \\
\hline Hyperlipidemia & $708(27.7)$ \\
\hline Ischemic heart disease & $107(4.2)$ \\
\hline Current smoking & $449(17.6)$ \\
\hline Systolic blood pressure $(\mathrm{mmHg})$ & $125 \pm 15$ \\
\hline Diastolic blood pressure $(\mathrm{mmHg})$ & $75 \pm 11$ \\
\hline HbAlc $(\%)$ & $5.9 \pm 0.8$ \\
\hline Fasting glucose $(\mathrm{mg} / \mathrm{dL})$ & $97 \pm 23$ \\
\hline Total cholesterol $(\mathrm{mg} / \mathrm{dL})$ & $199 \pm 36$ \\
\hline White blood cell $\left(\times 10^{3} / \mu \mathrm{L}\right)$ & $5.46 \pm 1.63$ \\
\hline Erythrocyte sedimentation rate $(\mathrm{mm} / \mathrm{hours})$ & $12 \pm 13$ \\
\hline High-sensitivity C-reactive protein $(\mathrm{mg} / \mathrm{dL})$ & $0.17 \pm 0.67$ \\
\hline Anti-platelet agent use & $320(12.5)$ \\
\hline White matter hyperintensity $(\mathrm{mL})$ & $2.53 \pm 6.06$ \\
\hline Silent brain infarct & $193(7.6)$ \\
\hline Cerebral microbleeds & $108(4.2)$ \\
\hline Values are presented as number & \\
\hline
\end{tabular}

Values are presented as number (\%) or mean \pm standard deviation. 
TABLE 2. Univariate linear regression analysis between white matter hyperintensity volume and demographic, clinical, laboratory, and radiological factors

\begin{tabular}{lcc}
\hline & $\beta$ changes in square root of WMH volume $(95 \% \mathrm{Cl})$ & $p$-value \\
\hline Age (years) & $0.049(0.045$ to 0.053$)$ & $<0.001$ \\
\hline Sex (male) & $0.011(-0.074$ to 0.095$)$ & 0.804 \\
\hline Body mass index $\left(\mathrm{kg} / \mathrm{m}^{2}\right)$ & $0.001(-0.014$ to 0.014$)$ & 0.998 \\
\hline Hypertension & $0.445(0.351$ to 0.540$)$ & $<0.001$ \\
\hline Diabetes & $0.391(0.277$ to 0.505$)$ & $<0.001$ \\
\hline Hyperlipidemia & $0.097(0.003$ to 0.191$)$ & 0.042 \\
\hline Ischemic heart disease & $0.122(-0.087$ to 0.332$)$ & 0.252 \\
\hline Current smoking & $-0.187(-0.297$ to -0.077$)$ & 0.001 \\
\hline Systolic blood pressure $(\mathrm{mmHg})$ & $0.009(0.007$ to 0.012$)$ & $<0.001$ \\
\hline Diastolic blood pressure $(\mathrm{mmHg})$ & $0.006(0.002$ to 0.010$)$ & 0.002 \\
\hline HbAic $(\%)^{*}$ & $0.851(0.575$ to 1.128$)$ & $<0.001$ \\
\hline Fasting glucose, $\mathrm{mg} / \mathrm{dL}{ }^{*}$ & $0.126(0.086$ to 0.166$)$ & $<0.001$ \\
\hline Total cholesterol $(\mathrm{mg} / \mathrm{dL})$ & $-0.002(-0.003$ to 0.000$)$ & 0.008 \\
\hline White blood cell $(\mathrm{xiO} / \mu \mathrm{L})$ & $0.051(0.025$ to 0.076$)$ & $<0.001$ \\
\hline Erythrocyte sedimentation rate $\left(\mathrm{mm} /\right.$ hours $^{*}$ & $0.078(0.052$ to 0.104$)$ & $<0.001$ \\
\hline High-sensitivity CRP $(\mathrm{mg} / \mathrm{dL})^{*}$ & $0.021(-0.121$ to 0.163$)$ & 0.771 \\
\hline Anti-platelet agent use & $0.323(0.197$ to 0.449$)$ & $<0.001$ \\
\hline
\end{tabular}

WMH; white matter hyperintensity, CRP; C-reactive protein.

*These variables were introduced as squared root scale.

TABLE 3. Multivariate linear regression analysis of possible predictors for white matter hyperintensity volume

\begin{tabular}{lcc}
\hline & $\beta$ changes in square root of WMH volume $(95 \% \mathrm{Cl})$ & $p$-value \\
\hline Age (years) & $0.046(0.042$ to 0.051$)$ & $<.001$ \\
Sex $($ male) & $0.055(-0.033$ to 0.143$)$ & 0.219 \\
Body mass index $\left(\mathrm{kg} / \mathrm{m}^{2}\right)$ & $-0.008(-0.021$ to 0.004$)$ & 0.203 \\
Hypertension & $0.187(0.092$ to 0.281$)$ & $<0.001$ \\
Diabetes & $0.093(-0.015$ to 0.201$)$ & 0.091 \\
Hyperlipidemia & $-0.053(-0.139$ to 0.033$)$ & 0.226 \\
Current smoking & $0.008(-0.102$ to 0.118$)$ & 0.893 \\
White blood cell $\left(x 10^{3} / \mu \mathrm{L}\right)$ & $0.042(0.018$ to 0.067$)$ & 0.001 \\
Erythrocyte sedimentation rate $(\mathrm{mm} /$ hours) & $0.027(0.001$ to 0.053$)$ & 0.043 \\
Anti-platelet agent & $-0.002(-0.121$ to 0.118$)$ & 0.978 \\
\hline
\end{tabular}

WMH; white matter hyperintensity.

*These variables were introduced as squared root scale.

be more closely related to inflammatory pathology than other cSVD subtypes.

The exact mechanisms explaining the close relationship between ESR and WMH volume are unclear. However, we suggested several plausible hypotheses. First, it may be due to the destruction of the blood-brain barrier (BBB) by endothelial dysfunction. Inflammatory cytokines and reactive oxygen species arising from subclinical inflammation conditions can interfere with the secretion of nitric oxide by endothelial cells. ${ }^{19,21}$ Alternatively, the BBB itself may be mechanically damaged by an upregulated inflammatory cascade. ${ }^{19,22}$ Damaged 
TABLE 4. Univariate linear regression analysis between erythrocyte sedimentation rate and demographic, clinical, and laboratory parameters

\begin{tabular}{|c|c|c|}
\hline & $\beta$ changes in square root of ESR $(95 \% \mathrm{CI})$ & $p$-value \\
\hline Age (years) & $0.036(0.029$ to 0.042$)$ & $<0.001$ \\
\hline Sex (male) & $-1.235(-1.351$ to -1.119$)$ & $<0.001$ \\
\hline Body mass index $\left(\mathrm{kg} / \mathrm{m}^{2}\right)$ & $-0.020(-0.040$ to 0.000$)$ & 0.055 \\
\hline Hypertension & $0.072(-0.071$ to 0.215$)$ & 0.324 \\
\hline Diabetes & $0.312(0.141$ to 0.483$)$ & $<0.001$ \\
\hline Hyperlipidemia & 0.167 (0.027 to 0.306$)$ & 0.019 \\
\hline Ischemic heart disease & $-0.004(-0.316$ to 0.308$)$ & 0.978 \\
\hline Current smoking & $-0.539(-0.702$ to -0.376$)$ & $<0.001$ \\
\hline Systolic blood pressure (mmHg) & $-0.003(-0.007$ to 0.001$)$ & 0.173 \\
\hline Diastolic blood pressure $(\mathrm{mmHg})$ & $-0.013(-0.018$ to -0.007$)$ & $<0.001$ \\
\hline $\operatorname{HbAlc}(\%)^{*}$ & $1.214(0.802$ to 1.626$)$ & $<0.001$ \\
\hline Fasting glucose $(\mathrm{mg} / \mathrm{dL})^{*}$ & 0.103 (0.044 to 0.162$)$ & 0.001 \\
\hline Total cholesterol (mg/dL) & $0.003(0.001$ to 0.005$)$ & 0.001 \\
\hline White blood cell $\left(\mathrm{x} \mathrm{O}^{3} / \mu \mathrm{L}\right)$ & $-0.003(-0.041$ to 0.035$)$ & 0.872 \\
\hline High-sensitivity CRP $(\mathrm{mg} / \mathrm{dL})^{*}$ & 1.621 (1.419 to 1.822$)$ & $<0.001$ \\
\hline Anti-platelet agent use & $-0.022(-0.211$ to 0.167$)$ & 0.820 \\
\hline
\end{tabular}

ESR; erythrocyte sedimentation rate, CRP; C-reactive protein.

*These variables were introduced as squared root scale.

$\mathrm{BBB}$, functionally or mechanically, causes periventricular infiltration of several toxic materials (e.g., protease, immunoglobulin, and complement components), and can cause WMH by damaging the surrounding neural tissues. ${ }^{8,19,23}$ In addition, damaged BBB can prevent clearance of interstitial fluid through the glymphatic pathway, which can also lead to WMH., ${ }^{1,}$ In our data, not only ESR but also elevated WBC counts showed a positive correlation with WMH volume, so this hypothesis seems valid. Second, chronic diffuse hypoperfusion may have been involved. The aforementioned decrease in nitric oxide production of endothelium can also decrease the ability to control cerebral perfusion through autoregulation. ${ }^{23,24}$ In addition, subclinical inflammation may prevent the proliferation of vascular smooth muscle cells and proceeds to fibrosis, causing atherosclerosis of small arterioles permanently. ${ }^{23,25,26}$ This is evidenced by several results showing that ESR is closely related to arterial stiffness in previous studies. ${ }^{15}$ In addition to small vessels, ESR has been shown to be closely correlated with atherosclerosis of large vessels. ${ }^{11,12}$ Chronic diffuse hypoperfusion caused by the stenosis of such large and small vessels is sufficient to grow
WMH volumes. Last, it may simply be the result of ESR and WMH sharing multiple risk factors. Of course, subclinical inflammation is known to be closely related to several metabolic diseases. ${ }^{27}$ However, in our data, ESR showed a close correlation with older age, diabetes, hyperlipidemia, high BP, hs-CRP level, but not with WBC counts. Therefore, although it has not yet been identified, there may be a link between them through pathway other than the inflammation.

Interestingly, ESR showed a close association only with WMH and no statistical significance with SBI or CMBs. This phenomenon is interpreted as the fact that other pathological mechanisms unrelated to subclinical inflammation and endothelial dysfunction play many roles in the development of SBI and CMBs. In addition to lipohyalinosis, it is known that embolism caused by proximal sources and microatheroma are involved in SBI development. ${ }^{3,7}$ In case of CMBs, lobar and deep CMBs are caused by different mechanisms, and hypertensive insult or amyloid angiopathy can affect this. ${ }^{1,6}$ However, in addition to differences in mechanisms, these statistical results may have arisen due to the unusually low prevalence of SBI and CMBs in our young 
and healthy study population.

There are several caveats to interpret our results. First, this study is a retrospective cross-sectional design. Therefore, we can only prove the association between the ESR and the WMH volume, not the causal relationship. For this, further prospective study is needed. Second, we conducted a study on a relatively young and healthy population. Thus, the influence of age and various vascular risk factors may have been relatively underestimated, and the influence of ESR may have been overestimated. Third, in order to accurately measure the $\mathrm{WMH}$ volume, the whole brain volume should have been measured as well. By standardizing on the basis of the ratio between the two, it was possible to minimize the difference due to the head size. Fourth, ESR is a parameter that is affected by various medical conditions such as sex, obesity, menstruation, and medication. Therefore, when applied in practice, it must be judged comprehensively with the clinical situation or other biomarkers. Last, we measured the periventricular WMH and subcortical WMH together when rating the WMH volumes. In previous studies, different mechanisms and characteristics have been reported between the two areas of $\mathrm{WMH} .^{28}$ Due to technical limitations, this study could not separate the two. Interesting results can be obtained if we can examine the relationship between ESR and WMH in the periventricular and subcortical areas respectively.

In conclusion, we demonstrated that ESR was closely related to WMH volume in a healthy population. If we classify high-risk groups through ESR and manage each of them appropriately, it may be helpful in preventing the development of WMH. By doing so, we will be able to prevent the occurrence of several neurological disorders in the subclinical stage before symptoms develop. However, our findings are needed to be validated in further prospective studies.

\section{Conflicts of interest}

No potential conflicts of interest relevant to this article was reported.

\section{REFERENCES}

1. Wardlaw JM, Smith EE, Biessels GJ, Cordonnier C, Fazekas
F, Frayne R, et al. Neuroimaging standards for research into small vessel disease and its contribution to ageing and neurodegeneration. Lancet Neurol. 2013;12:822-838.

2. Shi Y, Wardlaw JM. Update on cerebral small vessel disease: a dynamic whole-brain disease. Stroke Vasc Neurol. 2016;1:8392.

3. Lee EJ, Kang DW, Warach S. Silent new brain lesions: innocent bystander or guilty party? J Stroke. 2016;18:38-49.

4. Appleton JP, Woodhouse LJ, Adami A, Becker JL, Berge E, Cala LA, et al. Imaging markers of small vessel disease and brain frailty, and outcomes in acute stroke. Neurology. 2020;94:e439-e452.

5. Rost NS, Rahman RM, Biffi A, Smith EE, Kanakis A, Fitzpatrick $\mathrm{K}$, et al. White matter hyperintensity volume is increased in small vessel stroke subtypes. Neurology. 2010;75:1670-1677.

6. Wardlaw JM, Smith C, Dichgans M. Mechanisms of sporadic cerebral small vessel disease: insights from neuroimaging. Lancet Neurol. 2013;12:483-497.

7. Pantoni L. Cerebral small vessel disease: from pathogenesis and clinical characteristics to therapeutic challenges. Lancet Neurol. 2010;9:689-701.

8. Wardlaw JM. Blood-brain barrier and cerebral small vessel disease. J Neurol Sci. 2010;299:66-71.

9. Yoshida M, Higashi K, Kobayashi E, Saeki N, Wakui K, Kusaka T, et al. Correlation between images of silent brain infarction, carotid atherosclerosis and white matter hyperintensity, and plasma levels of acrolein, IL-6 and CRP. Atherosclerosis. 2010;211:475-479.

10. Satizabal CL, Zhu YC, Mazoyer B, Dufouil C, Tzourio C. Circulating IL-6 and CRP are associated with MRI findings in the elderly: the 3C-Dijon Study. Neurology. 2012;78:720-727.

11. Erikssen G, Liestøl K, Bjørnholt JV, Stormorken H, Thaulow

E, Erikssen J. Erythrocyte sedimentation rate: a possible marker of atherosclerosis and a strong predictor of coronary heart disease mortality. Eur Heart J. 2000;21:1614-1620.

12. Natali A, L'Abbate A, Ferrannini E. Erythrocyte sedimentation rate, coronary atherosclerosis, and cardiac mortality. Eur Heart J. 2003;24:639-648.

13. Li C, Zhang Q, Wang Q, Zhong J, Wang L, Li K, et al. Prognostic values of the SYNTAX score II and the erythrocyte sedimentation rate on long-term clinical outcomes in STEMI patients with multivessel disease: a retrospective cohort study. BMC Cardiovasc Disord. 2020;20:213.

14. Chamorro A, Vila N, Ascaso C, Saiz A, Montalvo J, Alonso P, et al. Early prediction of stroke severity. Role of the eryth- 
rocyte sedimentation rate. Stroke. 1995;26:573-576.

15. De Silva DA, Woon FP, Gan HY, Chen C, Chang HM, Cameron J, et al. Arterial stiffness is associated with raised levels of the inflammatory marker erythrocyte sedimentation rate among ischaemic stroke patients. Intern Med J. 2008;38:918920.

16. Zhou J, Wu J, Zhang J, Xu T, Zhang H, Zhang Y, et al. Association of stroke clinical outcomes with coexistence of hyperglycemia and biomarkers of inflammation. J Stroke Cerebrovasc Dis. 2015;24:1250-1255.

17. Xie D, Hu D, Zhang Q, Sun Y, Li J, Zhang Y. Increased high-sensitivity C-reactive protein, erythrocyte sedimentation rate and lactic acid in stroke patients with internal carotid artery occlusion. Arch Med Sci. 2016;12:546-551.

18. Balestrino M, Partinico D, Finocchi C, Gandolfo C. White blood cell count and erythrocyte sedimentation rate correlate with outcome in patients with acute ischemic stroke. J Stroke Cerebrovasc Dis. 1998;7:139-144.

19. Nam KW, Kwon HM, Jeong HY, Park JH, Kim SH, Jeong SM, et al. High neutrophil to lymphocyte ratio is associated with white matter hyperintensity in a healthy population. $J$ Neurol Sci. 2017;380:128-131.

20. Nam KW, Kwon HM, Jeong HY, Park JH, Kim SH, Jeong $\mathrm{SM}$, et al. Cerebral white matter hyperintensity is associated with intracranial atherosclerosis in a healthy population. Atherosclerosis. 2017;265:179-183.

21. Abbott NJ. Inflammatory mediators and modulation of blood-brain barrier permeability. Cell Mol Neurobiol. 2000;20:131-147.

22. Varatharaj A, Galea I. The blood-brain barrier in systemic inflammation. Brain Behav Immun. 2017;60:1-12.

23. Hassan A, Hunt BJ, O'Sullivan M, Parmar K, Bamford JM, Briley D, et al. Markers of endothelial dysfunction in lacunar infarction and ischaemic leukoaraiosis. Brain. 2003;126:424-432.

24. Bakker SL, de Leeuw FE, de Groot JC, Hofman A, Koudstaal PJ, Breteler MM. Cerebral vasomotor reactivity and cerebral white matter lesions in the elderly. Neurology. 1999;52:578583.

25. Austin RC, Lentz SR, Werstuck GH. Role of hyperhomocysteinemia in endothelial dysfunction and atherothrombotic disease. Cell Death Differ. 2004;11 Suppl 1:S56-S64.

26. Cloonan L, Fitzpatrick KM, Kanakis AS, Furie KL, Rosand J, Rost NS. Metabolic determinants of white matter hyperintensity burden in patients with ischemic stroke. Atherosclerosis. 2015;240:149-153.

27. Mardi T, Toker S, Melamed S, Shirom A, Zeltser D, Shapira I, et al. Increased erythropoiesis and subclinical inflammation as part of the metabolic syndrome. Diabetes Res Clin Pract. 2005;69:249-255.

28. Lee KO, Woo MH, Chung D, Choi JW, Kim NK, Kim OJ, et al. Differential impact of plasma homocysteine levels on the periventricular and subcortical white matter hyperintensities on the brain. Front Neurol. 2019;10:1174. 